\title{
Clinical Outcomes of Synchronous Laparoscopic Cholecystectomy with Coronary Artery Revascularization
}

\author{
Dogan Kahraman, MD, ${ }^{1}$ Ihsan Sami Uyar, MD, ${ }^{2}$ Umit Duman, MD,${ }^{3}$ Ibrahim Sami Karaca, MD, ${ }^{2}$ \\ Dilek Dogan, PhD,${ }^{4}$ Kevser Tural, MD,${ }^{5}$ Omer Faruk Dogan, MD,${ }^{5}$ Atif Akcevin ${ }^{3}$ \\ ${ }^{1}$ Department of Cardiovascular Surgery, Gaziantep University School of Medicine, Gaziantep, Turkey; ${ }^{2}$ Department of Cardiovascular \\ Surgery, Kirsehir Ahi Evran University School of Medicine, Kirsehir, Turkey; ${ }^{3}$ Department of Cardiovascular Surgery, Koc Vakfi \\ American Hospital, Istanbul, Turkey; ${ }^{4}$ Department of Nutrition and Dietetics, The Health Science University, Adana City Hospital, \\ Adana, Turkey; ${ }^{5}$ Department of Cardiovascular Surgery, Kafkas University School of Medicine, Kars, Turkey
}

\section{ABSTRACT}

Background: There are limited data about the results of simultaneous coronary revascularization, either with coronary artery bypass grafting (CABG) surgery or percutaneous coronary intervention (PCI), and cholecystectomy operations. Here we present clinical outcomes of the patients who underwent simultaneous laparoscopic cholecystectomy (LC) and coronary revascularization at the same session.

Patients and Methods: We included a total of 19 patients who underwent simultaneous LC and CABG or PCI. Thirteen of them had been hospitalized because of acute cholecystitis prior to coronary angiography. Simultaneous CABG and LC were performed in 10 patients (group I). LC was performed immediately after CABG surgery at the same session. PCI (group II) was performed in 9 patients. In the PCI group, LC was performed under general anesthesia 2 or 3 days after PCI.

Results: No mortality was seen after the procedures. In the CABG group, the mean number of bypass grafts was $3.4 \pm 1.9$. The mean extracorporeal circulation and the total operation times were $95 \pm 13.5$ minutes and $259 \pm 18.9$ minutes, respectively; the mean intubation duration was $17 \pm$ 4.8 hours. In the PCI group, the mean number of stents per patient was $2.1 \pm 0.7$; LC was performed 2 or 3 days after the PCI without the cessation of clopidogrel and acetylsalicylic acid. The mean operation times for LC were $56.5 \pm$ 15.6 minutes and $51.3 \pm 17.6$ minutes in the CABG and PCI groups, respectively $(P=.86)$. In the $\mathrm{CABG}$ group, the mean durations of ICU and hospital stays were $3.1 \pm 1.4$ and 14.2 \pm 3.7 days, respectively. In the PCI group, the mean durations of ICU stay and hospitalization were $1.7 \pm 0.4$ and 7.4 \pm 2.2 days, respectively. Significant differences were found between the 2 groups in terms of the intubation time, duration of ICU stay, and hospitalization periods $(P=.001, P$

Received August 24, 2017; received in revised form fanuary 15, 2018; accepted March 7, 2019.

Correspondence: Omer Faruk Dogan, MD, Kafkas University School of Medicine, Department of Cardiovascular Surgery, 36001 Kars, Turkey; +90533-4813056; fax: +90-474-2250101 (e-mail: ofdogan@bacettepe.edu.tr).
$=.0001$, and $P=.001$, respectively). No intra-abdominal complications or bleeding was encountered in any group. Postoperative complications of the abdominal wall or mediastinitis were not seen in the setting of concomitant procedures in the CABG group.

Conclusion: Simultaneous CABG or PCI with LC may be performed safely in patients with cholecystitis. The durations of postcholecystectomy ICU stay and the intubation time were significantly lower in the PCI group. According to our results, PCI may be the first choice of revascularization procedure in selected patients requiring cholecystectomy prior to discharge.

\section{INTRODUCTION}

Cholelithiasis can be detected in patients with coronary artery disease (CAD) requiring revascularization. Fatal myocardial ischemia has been previously reported in a patient who underwent cholecystectomy by Lee et al [Lee 2010]. The rate of symptomatic acute cholelithiasis with the cholecystectomy indication has been reported in $0.5 \%-0.7 \%$ of CAD patients [Alvino 2017]. Unfortunately, there are inadequate data about simultaneous cholecystectomy and coronary revascularization. In addition, to our knowledge there is no report about simultaneous cholecystectomy in the early period after the percutaneous coronary intervention (PCI) in patients with cholelithiasis or choledocholithiasis. In limited clinical experiences, Kioke et al and Hekmet et al showed that the risk of myocardial infarction during an abdominal surgery might be reduced after the coronary artery bypass grafting (CABG) operation [Koike 1991; Hekmat 2005]. Treatment of cholecystitis may be performed in an elective or semi-elective situation after cardiac surgery. Because there is a limited amount of scientific information in the literature, no common consensus is present about the treatment methods of cholelithiasis in CAD patients [Bilge Erdogan 2017].

We conducted this study to assess the safety and efficacy of concomitant laparoscopic cholecystectomy (LC) immediately after the CABG and in the early time after the PCI in 2 centers. 


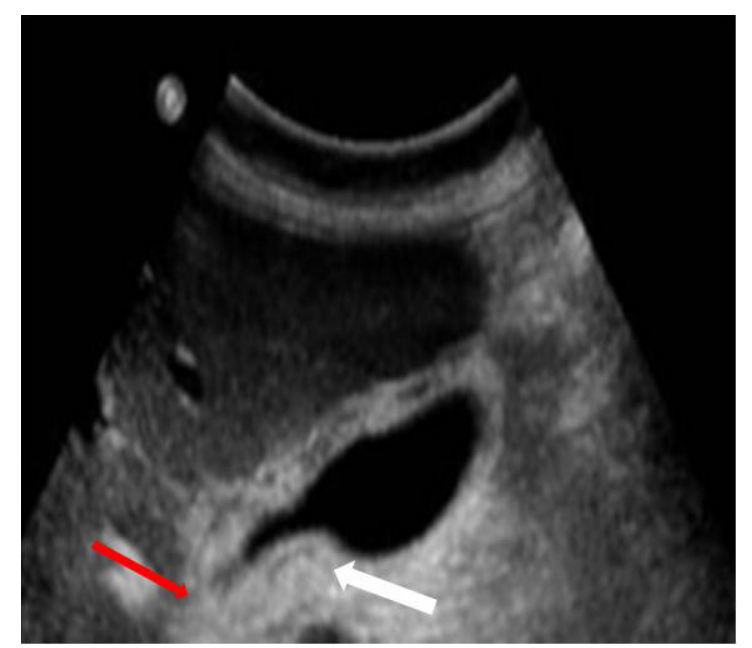

Figure 1. An abdominal ultrasonography is showing gall bladder and ductus choledochus. Red arrow shows a common bile duct. White arrow indicates the gall stone.

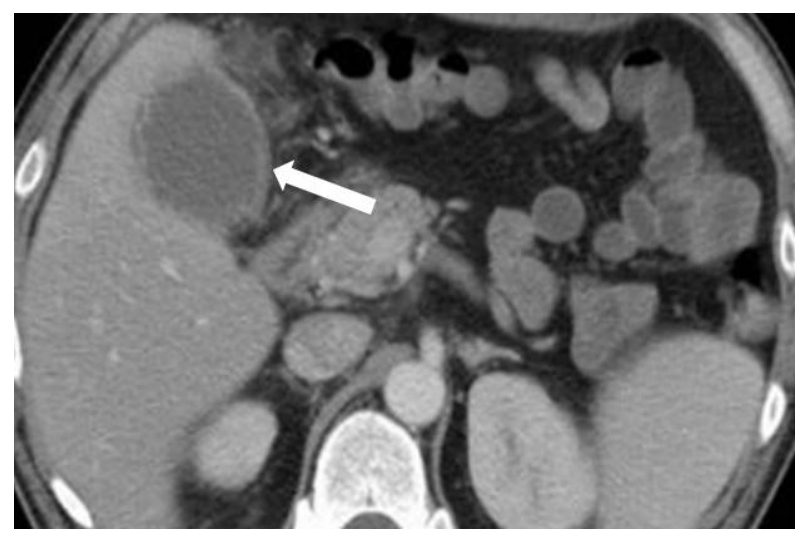

Figure 2. Abdominal enhanced computed tomography is showing gall bladder and liver in a coronary artery bypass grafting patient. White arrow indicates the edematous gall bladder.

\section{PATIENTS AND METHODS}

This study was carried out in accordance with the Declaration of Helsinki guidelines and was approved by the institutional review board in Koc Vakfi American Hospital, Istanbul, Turkey. All patients have given their written informed consent. The study included 19 patients with CAD and abdominal symptoms related to cholelithiasis or choledocholithiasis as confirmed by ultrasonography or abdominal computed tomography (Figures 1 and 2) between January 2008 and June 2016. LC was performed in all patients after coronary revascularization.

The mean age of the patients was $53.0 \pm 11.6$ years (49-72 years). The preoperative characteristics of the patients in both groups are given in Table 1. LC and CABG were simultaneously performed in group I (11 patients [57.8\%] with a mean age of $56 \pm 7.8$ years [ $48-71$ years). The CABG operations were performed using a beating heart technique in 4 patients;
Table 1. Preoperative Characteristics of Patients Who Underwent Open Heart Surgery with (Group I) or without (Group II) Concomitant Cholecystectomy*

\begin{tabular}{lccc}
\hline & No. of Patients & CABG & PCl \\
\hline & 19 & 11 & 8 \\
Male/female & $11 / 8$ & $7 / 4$ & $5 / 3$ \\
\hline & CABG & PCl & $P$ \\
\hline Characteristics & & & \\
Age (years) & $56 \pm 7.8$ & $49 \pm 9.8$ & .74 \\
BMl & $29.1 \pm 2.6$ & $28.2 \pm 1.8$ & .66 \\
Previous MI [n (\%)] & $4(36.3 \%)$ & $2(25 \%)$ & .76 \\
Three vessels[n (\%] & $8(72.7 \%)$ & $0(0 \%)$ & .000 \\
Two vessels[n(\%)] & $2(18.1 \%)$ & $3(37.5 \%)$ & .034 \\
One vessel[n (\%)] & $1(9 \%)$ & $5(62.5 \%)$ & .0001 \\
Symptoms & & & \\
Asymptomatic (n) & 3 & 2 & \\
Hypochondralgia(n) & 6 & 5 & \\
Jaundice(n) & 2 & 1 & \\
Dilated common bile duct(n) & 4 & 3 & \\
Elevated liver enzyme(n) & 2 & 1 & \\
\hline
\end{tabular}

*CABG, coronary artery bypass grafting; $\mathrm{PCl}$, percutaneous coronary intervention,BMI: body mass index; MI,myocardial infarction.

the PCI was performed using plain old balloon angioplasty (POBA) or drug-eluting stent (DES) ingroup II (8 patients with a mean age of $49 \pm 9.8$ years [ $46-72$ years]). In this group, the LC was performed under general anesthesia 2 or 3 days after coronary stenting. The main indications for cholecystectomy were history of gallstone pancreatitis, jaundice, elevated liver enzymes, right hypochondralgia, or a dilated common bile duct. There was only one patient with a history of abdominal surgery because of gastric cancer.

\section{Simultaneous $C A B G$ and $L C$}

After a midline sternotomy, the left internal mammary artery (LIMA) and saphenous vein were harvested. Heparin (3 IU/kg) was given, and an extracorporeal circulation (ECC) was instituted via ascending aorta and a single venous cannulation. After aortic cross-clamping, myocardial arrest was provided using an antegrade and a retrograde cold blood cardioplegias. At the end of distal anastomosis, proximal anastomosis was done using an aortic side clamp. We performed coronary revascularization using a beating heart technique using intracoronary shunt in 4 patients. After CABG, heparin effect was neutralized using protamine, and meticulous hemostasis was achieved carefully. After the closure of sternotomy, the LC was performed without any complication. No significant hemodynamic instability was detected during the LC. 
Table 2. Peri- and Postoperative Characteristics of Both Groups

\begin{tabular}{lccc}
\hline & CABG & PCl & $P$ \\
\hline Operation time (minutes) & $259 \pm 18.9$ & $59 \pm 18.9$ & .000 \\
Intubation time (minutes) & $17 \pm 9.6$ & $5.2 \pm 9.6$ & .001 \\
ICU stay (days) & $3.1 \pm 1.4$ & $1.7 \pm 0.4$ & .0001 \\
Hospitalisation (days) & $14.2 \pm 3.7$ & $7.4 \pm 2.2$ & .001 \\
Number of vessels & $3.4 \pm 1.9$ & $2.1 \pm 0.9$ & .034
\end{tabular}

\section{PCI and LC}

Exclusion criteria were emergent PCI, history of thrombolytic agent infusion, left ventricular ejection fraction $<30 \%$, left main coronary artery disease, hematologic disorders, and chronic kidney disease. PCI or POBA were used (Figure 3). The patients were treated with a loading dose of $100 \mathrm{mg}$ aspirin and $300 \mathrm{mg}$ clopidogrel before the procedure, followed by maintenance dual antiplatelet therapy $(75 \mathrm{mg}$ clopidogrel and $100 \mathrm{mg}$ ASA, daily). Two or 3 days later, the LC was performed uneventfully.

\section{$L C$}

After a subcostal mini-incision, a minimal amount of carbon dioxide insufflation was performed to inhibit respiratory problems resulting from the diaphragmatic elevation. The LC was performed using the standard method. There was no intra-abdominal or pulmonary complication. A 7-day course of antibiotics was given after the LC. Oral feeding was started the day after extubation. In the PCI group, dual antiplatelet therapy was started on postoperative day 1 . The demographic characteristics including preoperative risk factors, surgical data, and the lengths of ICU and hospital stay were recorded for both groups. The operative and postoperative data are summarized in Table 2.

\section{Statistical Analyses}

All statistical analyses were conducted using SPSS, version 18.0 (PASW Statistics for Windows; SPSS Inc., Chicago, IL, USA). Descriptive statistical methods were used to describe the data. Results were presented as mean \pm standard deviation for continuous variables and frequency (percentages) for categorical variables. The comparisons between 2 groups were performed using an unpaired $\mathrm{t}$ test for continuous variables and Pearson $\chi^{2}$ test for categorical variables. All tests were 2 -sided, and results with a $P$ value $<.05$ were considered statistically significant.

\section{RESULTS}

The complications such as myocardial ischemia, stroke, renal insufficiency, and pulmonary complications did not occur after the operations or PCI. In one patient from the CABG group, the duration of intubation and ICU stay was prolonged because of respiratory failure. The mean duration of associated procedures was $298.9 \pm 33.9$ minutes in this

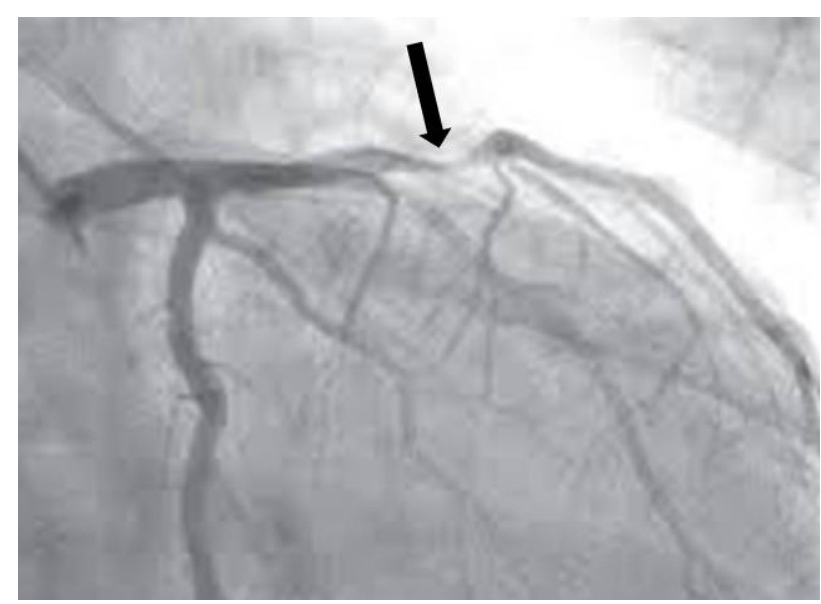

Figure 3. A coronary artery angiographic image is showing the left main coronary artery and its branches such as the left anterior descending and circumflex artery prior to percutanous coronary artery intervention. Black arrow indicates the severe occlusion of the left anterior descending artery with the circumflex branch of the left main stem.

group. Durations of LC in the CABG and PCI groups were $56.5 \pm 15.6$ minutes and $51.3 \pm 17.6$ minutes, respectively $(P>.05)$. The mean intubation duration was $17 \pm 9.6$ hours and $5.2 \pm 9.6$ hours after the CABG and PCI, respectively $(P=.001)$. The mean ICU stay time was $3.1 \pm 1.4$ days in the CABG group and $1.7 \pm 0.4$ days in the PCI group $(P=.0001)$. The mean duration of hospital stay was $14.2 \pm 3.7$ days in the CABG group and $7.4 \pm 2.2$ days in the PCI group $(P=.001)$.

No intra-abdominal complications were seen in either group. We have not detected wound infection in abdominal incision. No electrocardiographic change such as ST elevation or new $\mathrm{Q}$ wave was seen in either group. The postoperative course was uneventful.

\section{DISCUSSION}

There is no common consensus about the treatment method in patients with cholecystitis who have severe CAD requiring coronary revascularization. The first case of simultaneous LC and CABG was been reported in 1989 by Hoshino et al [Hoshino 1989]. In 2002, successful clinical results of concomitant CABG and cholecystectomy were reported in the largest case series ( $\mathrm{n}=55$ patients) by Hirose et al [Hirose 2002]. Charokopos et al carried out simultaneous cholecystectomy (open or laparoscopic) and open heart surgery in 7 selected patients without any complication [Charokopos 2007]. Concomitant cholecystectomy and open heart surgery have been reported by Hirose and colleagues and Charokopos et al in 2002 and in 2007, respectively [Hirose 2002; Charokopos 2007]. The first case of simultaneous LC and CABG was performed in 1989 by Hoshino et al [Hoshino 1989]. In accordance with previous publications, simultaneous LC and CABG surgery could be performed in selected patients [Hirose 2002; Bilge Erdogan 2017]. 
Because the gallbladder has an edematous wall that is fragile early on in acute cholecystitis, Charokopos et al and Hekmat and colleagues have suggested the drainage of the gallbladder under local anesthesia and preferred to postpone cholecystectomy to after CABG. Concomitant surgery of LC and CABG may sound aggressive but could be done safely by experienced surgeons, and it may be advantageous for patients to avoid complications of delayed abdominal surgery [Hirose 2002; Hekmat 2005; Charokopos 2007; Bilge Erdogan 2017].

It is known that gastrointestinal complications including acalculous cholelithiasis may be encountered 5 or 7 days after CABG, presenting with pain and tenderness on the right hypocondrium with or without liver dysfunction [Ohri 1991; Mangi 2005; Charokopos 2007; Yang 2016]. The mortality rate of cholelithiasis or choledocholithiasis was reported as high as $15 \%$ to $42 \%$ of these patients [Yang 2016; Mangi 2005]. Urgent cholecystectomy may require general anesthesia shortly after CABG, which may lead to life-threatening complications such as intraoperative hypotension or myocardial ischemia. Therefore, simultaneous cholecystectomy and coronary revascularization is believed to save the patient from the additional general anesthesia and abdominal surgery needed for the separate cholecystectomy and from the complications associated with them.

The clinical outcome of the present cases is similar to previous publications, but the present study has one significant difference. To the best of our knowledge, the present study is the first to demonstrate that the LC can safely be performed 2-3 days after the PCI without the cessation of antiplatelets. It is crucial to use dual antiplatelet therapy after coronary stenting for 12 months as recommended in current guidelines, regardless of the type of stent [Perugini 1997]. The cessation of clopidogrel and ASA was suggested 5-7 days before abdominal surgical procedures [Levine 2011], though some authors suggested that perioperative mortality risk does not differ by cessation of antiplatelets within 7 days preoperatively [Chernoguz 2011; Helft 2016]. There is no randomized controlled clinical trial to clarify this issue, and a consensus is yet to be established in the management of such patients. Thus, some surgeons refuse to operate on these patients because of increased risk of bleeding complication. Recently, Benahmed et al showed that dual antiplatelet therapy was associated with a low risk of bleeding during abdominal surgery and does not seem to be a contraindication for LC [Benahmed 2014]. In a randomized prospective clinical trial on this particular patient group, Chernoguz et al reported that they had neither perioperative mortality nor bleeding complication after such a surgical procedure [Chernoguz 2011]. No intra-abdominal bleeding or stent thrombosis was seen in our PCI patients.

There is no adequate data about the clinical outcome of patients with cholecystitis who underwent simultaneous cholecystectomy and the CABG operation. Yang et al suggested using simultaneous cardiac and extracardiac operation in 14 patients, and they have performed concomitant cholecystectomy and CABG in 3 of 14 patients successfully [Yang 2016]. They have reported common bile duct exploration and T-tube drainage in one case. In accordance with the results of previous investigations [Hoshino 1989; Koike 1991; Hirose 2002; Hekmat 2005; Bilge Erdogan 2017], our results support the idea that simultaneous LC and coronary artery revascularization may be safely performed in selected patients by an experienced team. Because simultaneous surgery is an aggressive treatment method, Charokopos et al, Hekmat et al, Hirose et al, Hoshino et al, and Koike et al do not recommend it as a routine method for all patients [Hoshino 1989; Koike 1991; Hirose 2002; Hekmat 2005; Charokopos 2007]. On the other hand, simultaneous surgery may provide some benefits with favorable clinical outcomes in selected patients. Because management of high-risk patients is a difficult dilemma, some authors suggested a percutaneous cholecystostomy as a treatment of choice [Lee 2010]. LC, if performed by experienced surgeons, is associated with shorter hospital stay, low morbidity and mortality rate, and a good cosmetic result. Bilge Erdogan et al have reported successful results of simultaneous LC and CABG in a recent publication [Hekmat 2005]. It is really a challenging decision to manage $\mathrm{CAD}$ patients who need abdominal surgery at the same session.

Though both 1-stage and 2-stage procedures are possible, there are several arguments for and against both of these treatment strategies. In accordance with our experiences, we suggest simultaneous coronary artery revascularization (CABG or PCI) and cholecystectomy may be performed safely in selected patients by experienced clinics. Because we do not have a large number of patients or any case-control study, we do not suggest concomitant surgery in each patient. A 2-stage procedure means the administration of anesthetic agents twice for the CABG patients, doubles the surgical trauma, and results in a longer overall stay in hospital. Additionally, it duplicates the preoperative stress and postoperative pain and leads to higher costs of treatment.

In our opinion, we need a large number of randomized clinical trials for confirming the procedure of choice for the best clinical outcomes in these patients. The simultaneous operation might be performed in patients with a history of acute attack of chronic cholelithiasis or those who suffer from jaundice with dilated common bile duct and from recurrent pancreatitis with concomitant severe CAD. Performing simultaneous surgeries on patients with both cardiac and cholelithiasis is possible and acceptable considering the in-hospital mortality of $0 \%$ as reported by other researchers [Hoshino 1989; Hekmat 2005; Bilge Erdogan 2017]. There were no postoperative cardiac or abdominal events in both groups in the long-term follow-up of our patients.

\section{Conclusion}

Although simultaneous LC and coronary revascularization is an aggressive surgical approach, it may provide good prognosis if performed by an experienced team in carefully selected patients. The 3 -year survival rate was $\geq 87 \%$, and the clinical outcome of our limited number of patients was quite favorable. We believe that a complete preoperative evaluation and an accurate estimation of combined perioperative risk should be carried out before treatment planning in order to select the best surgical strategy in these patients. Our study will continue to collect data and summarize the efficacy of the simultaneous cholecystectomy and cardiac surgery. 


\section{REFERENCES}

Alvino DML, Fong ZV, McCarthy CJ, et al. 2017. Long-term outcomes following percutaneous cholecystostomy tube placement for treatment of acute calculous cholecystitis. J Gastrointest Surg 21(5):761-9.

Benahmed A, Kianda M, Ghisdal L, et al. 2014. Ticlopidine and clopidogrel, sometimes combined with aspirin, only minimally increase the surgical risk in renal transplantation: a case-control study. Neprol Dial Transplant 29(2):463-6.

Bilge Erdogan M, Kaplan M, Kazaz H, Salman B. 2017. Synchronous open heart surgery and laparoscopic cholecystectomy: an observational case study with 28 patients. Am Surg 83(3):314-21.

Charokopos N, Antonitsis P, Spanos C, Rouska E, Spanos P. 2007. Concomitant cholecystectomy and open heart surgery. Surg Today 37(8):638-41.

Chernoguz A, Telem DA, Chu E, Ozao-Choy J, Tammaro Y, Divino CM. 2011. Cessation of clopidogrel before major abdominal procedures. Arch Surg 146(3):334-9.

Hekmat M, Taghipoor HR, Nobahar MR, et al. 2005. Laparoscopic cholecystectomy and open-heart surgery at the same time. J Card Surg 20(6):557-9.

Helft G. 2016. Dual antiplatelet therapy duration after drug-eluting stents: how long? J Thorac Dis 8(8):E844-6.

Hirose H, Amano H, Takahashi A. 2002. Concomitant cholecystectomy and coronary artery bypass. Ann Thorac Cardiovasc Surg 8(6):358-62.
Hoshino R, Okubo T, Kaneko K, Gushiken S, Sato M. 1989. [Concomitant coronary artery bypass and cholecystectomy: a case report]. Kyobu Geka 42(12):1043-6. Japanese

Koike R, Kimura H, Sugihara J, et al. 1991. [Simultaneous coronary artery bypass grafting and cholecystectomy: a report of three cases]. Kyobu Geka 44(2):145-50. Japanese.

Lee JJ, Hwang SM, Kim HS, et al. 2010. Acute fatal postoperative myocardial infarction after laparoscopic cholecystectomy in a cardiac patient: a case report. Korean J Anesthesiol 59(suppl):S110-3.

Levine GN, Bates ER, Blankenship JC, et al. 2011. 2011 ACCF/AHA/ SCAI guideline for percutaneous coronary intervention: a report of the American College of Cardiology Foundation/American Heart Association Task Force on Practice Guidelines and the Society for Cardiovascular Angiography and Interventions. Circulation 124(23):e574-651.

Mangi AA, Christison-Lagay ER, Torchiana DF, Warshaw AL, Berger DL. 2005. Gastrointestinal complications in patients undergoing heart operation: an analysis of 8709 consecutive cardiac surgical patients. Ann Surg 241(6):895-901; discussion 901-4.

Ohri SK, Desai JB, Gaer JAR, et al. 1991. Intraabdominal complications after cardiopulmonary bypass. Ann Thorac Surg 52(4):826-31.

Perugini RA, Orr RK, Porter D, Dumas EM, Maini BS. 1997. Gastrointestinal complications following cardiac surgery:an analysis of 1477 cardiac surgery patients. Arch Surg 132(4):352-7.

Yang Y, Xiao F, Wang J, et al. 2016. Simultaneous surgery in patients with both cardiac and noncardiac diseases. Patient Prefer Adherence 10:1251-8 